\title{
Viscoelastic Buckling and Postbuckling of Circular Cylindrical Laminated Shells in Hygrothermal Environment
}

N.N. Huang

Department of Mechanical and Marine Engineering National Taiwan Ocean University Keelung 20224, Taiwan, Republic of China

Follow this and additional works at: https://jmstt.ntou.edu.tw/journal

Part of the Mechanical Engineering Commons

\section{Recommended Citation}

Huang, N.N. (1994) "Viscoelastic Buckling and Postbuckling of Circular Cylindrical Laminated Shells in Hygrothermal Environment," Journal of Marine Science and Technology. Vol. 2: Iss. 1, Article 2.

DOI: $10.51400 / 2709-6998.2483$

Available at: https://jmstt.ntou.edu.tw/journal/vol2/iss1/2

This Research Article is brought to you for free and open access by Journal of Marine Science and Technology. It has been accepted for inclusion in Journal of Marine Science and Technology by an authorized editor of Journal of Marine Science and Technology. 
Viscoelastic Buckling and Postbuckling of Circular Cylindrical Laminated Shells in Hygrothermal Environment

Acknowledgements

The support of this research by the National Science Council of Taiwan, R.O.C. through Grant NSC82-0401-E-019-18 is gratefully acknowledged.

This research article is available in Journal of Marine Science and Technology: https://jmstt.ntou.edu.tw/journal/vol2/ 


\title{
VISCOELASTIC BUCKLING AND POSTBUCKLING OF CIRCULAR CYLINDRICAL LAMINATED SHELLS IN HYGROTHERMAL ENVIRONMENT
}

\author{
N.N. Huang \\ Department of Mechanical and Marine Engineering \\ National Taiwan Ocean University \\ Keelung 20224, Taiwan, Republic of China
}

\begin{abstract}
Key words: viscoelastic buckling, postbuckling, HTSM, cylindrical laminated shell.
\end{abstract}

\begin{abstract}
The viscoelastic buckling and postbuckling behaviors of fiber-reinforced plastic (FRP) circular cylindrical shells, operating in the hygrothermal environment, subjected to uniform external pressure are investigated in this study. The FRP is assumed to be a hygrothermorheologically simple material (HTSM). The analysis is conducted within the framework of the quasi-elastic approach. The finite element method is employed to examine both buckling and postbuckling behaviors for shells having arbitrary lay-ups. The arc-length algorithm is incorporated into the finite element formulations to trace the postlimit equilibrium path. The structural responses of Glass/Epoxy shells are reported.
\end{abstract}

\section{INTRODUCTION}

The pressure vessels made of fiber-reinforced plastics (FRP) have been increasingly used in various areas, for examples, in marine and chemical industries. The excellent property against corrosion and relatively low cost in fabrication and maintenance make the FRP pressure vessels more favorable in some applications than the pressure vessels made of stainless steel or titanium alloy.

In this study, the viscoelastic buckling and postbuckling of FRP circular cylindrical shells, operating in the hygrothermal environment, subjected to uniform external pressure are investigated. It is known that the plastic matrix of FRP possesses a viscoelastic behavior. Therefore, FRP shells might suffer a precarious creep buckling when subjected to external pressure or vacuum. Pressure vessels frequently operate in hot and humid environment; the elevated hygrothermal conditions not only induce deformations in the shells but also accelerate the creep effect on the buckling. Because it is almost impossible to provide lifetime prototype creep buckling tests, rigorous creep analyses are seen to be very important for designing FRP shells under external pressure. Most researches on the buckling of composite shells are based on the elastic analysis. To the author's knowledge, only Chang and Cheng (1991) examined the creep buckling of simply-supported cross-ply cylindrical shells using a higher-order shell theory. Their analysis was based on the quasi-elastic approximation and neglected the prebuckling effect.

In the design of lightweight thin walled cylindrical shells, the determination of the buckling load alone is not sufficient in general, but it is further required to clarify the postbuckling behavior. One of the reason is to estimate the effect of practically unavoidable imperfections on the critical load. It has been known that in the buckling of thin walled cylinders, large discrepancies exist between theoretical and experimental results. The shell can be in equilibrium under a load much lower than the initial buckling load, indicating a significant reduction in critical load when imperfection exists. The experimental works on the buckling of isotropic shells under external pressure performed by Yamaki (1984) reveal that the critical load can be as low as the minimum postbuckling pressure.

The uniform external pressure and hygrothermal forces applied to the shells are considered to be timewise constant. For the sake of computational efficiency, yet without sacrificing a significant degree of accuracy, the 
shell analysis is conducted within the framework of the quasi-elastic approach (Wilson and Vinson, 1984). The finite element scheme, based on the first order shear deformation shell theory (FSDT), is employed to examine the structural responses of shells having arbitrary layups. The arc-length algorithm is incorporated into the finite element formulation to trace the limit point and snap-through point encountered along the postbuckling equilibrium path. The instant snap-through behavior is analyzed using the elastic approach; that is, no time elapse is assumed during the process of the snapping.

The FRP lamina is assumed to be a hygrothermorheologically simple material (HTSM). The effects of temperature and moisture concentration on the creep behavior are accommodated in the constitutive equation by the use of "reduced time". The numerical results of antisymmetric angle-ply and cross-ply glass reinforced epoxy (Glass/Epoxy) shells are presented.

\section{GOVERNING SHELL FORMULATIONS}

Consider an FRP circular cylindrical shell as shown in Fig. 1. The shell has thickness $h$, radius $R$ and length $L$. The middle surface of the shell is defined by $x$ and $y$ coordinates. The third coordinate $z$ is in the thickness direction. The laminated shell is constructed of an arbitrary number $(N)$ of orthotropic layers with the principal material directions of orthotropy oriented at some arbitrary angle with respect to the $x$ and $y$ coordinates of the shell. The shell is subjected to uniform external pressure $p$ as shown in Fig. 1. The variations of temperature and content of moisture concentration of the shell are denoted as $\Delta T$ and $\Delta c$, respectively.

According to the first order shear deformation theory (FSDT), the displacements $(\hat{u}, \hat{v}, \hat{w})$ of an arbitrary point in the shell are expressed as (Baker, 1968)

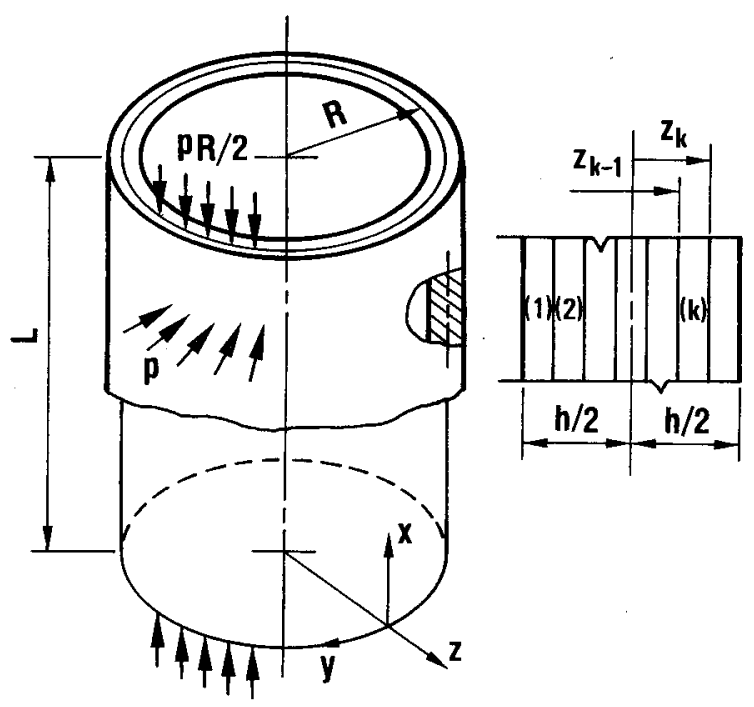

Fig. 1. Laminated circular cylindrical shell.

$$
\begin{aligned}
\hat{u}(x, y, z) & =u(x, y)-z \theta_{x}(x, y) \\
\hat{v}(x, y, z) & =v(x, y)-z \theta_{x}(x, y) \\
\hat{w}(x, y, z) & =w(x, y)
\end{aligned}
$$

where $u, v$ and $w$ are the middle surface displacements in the $x, y$ and $z$ directions, and $\theta_{x}$ and $\theta_{y}$ denote rotations of the cross sections perpendicular to the $x$ and $y$ coordinates, respectively.

The strains and curvatures of the middle surface are related to the displacements and rotations by

$$
\begin{aligned}
& \varepsilon_{x}=u_{, x}+\left(w_{, x}\right)^{2} / 2 \\
& \varepsilon_{y}=v_{, y}+w / R+\left(w_{, y}\right)^{2} / 2 \\
& \gamma_{x y}=v_{, x}+u_{, y}+w_{, x} w_{, y} \\
& \gamma_{y z}=w_{, y}-v / R-\theta_{y} \\
& \gamma_{x z}=w_{, x}-\theta_{x} \\
& \kappa_{x}=-\left(\theta_{x}\right)_{, x} \\
& \kappa_{y}=-\left(\theta_{y}\right)_{, y} \\
& \kappa_{x y}=-\left(\theta_{y}\right)_{, x}-\left(\theta_{x}\right)_{, y}
\end{aligned}
$$

in which a comma denotes differentiation with respect to the subscript. It is noted that the inclusion of the nonlinear terms is due to the von Karman assumption. For the case of linear analysis, these nonlinear terms are discarded.

The stress resultants are related with strains and curvatures, in contracted notations, as

$$
\begin{aligned}
& N_{i}=A_{i j} \varepsilon_{j}+B_{i j} \kappa_{j}-N_{i}^{T M} \quad(i, j=1,2,6) \\
& M_{i}=B_{i j} \varepsilon_{j}+D_{i j} \kappa_{j}-M_{i}^{T M} \quad(i, j=1,2,6)
\end{aligned}
$$

and

$$
Q_{m}=S_{m n} \varepsilon_{n} \quad(m, n=4,5)
$$

Here $A_{i j}, B_{i j}, D_{i j}$ and $S_{m n}$ are extensional, coupling, bending and transverse shear stiffnesses, and are defined by

$$
\begin{aligned}
& \left(A_{i j}, B_{i j}, D_{i j}\right)=\sum_{k=1}^{N} \int_{z_{k-1}}^{z_{k}}\left(1, z, z^{2}\right)\left(\bar{Q}_{i j}\right)_{k} \mathrm{~d} z \quad(i, j=1,2,6) \\
& S_{m n}=K_{m n} \sum_{k=1}^{N} \int_{z_{k-1}}^{z_{k}}\left(\bar{Q}_{m n}\right)_{k} \mathrm{~d} z(m, n=4,5 ; \text { not summed })
\end{aligned}
$$

in which $\left(\bar{Q}_{i j}\right)_{k}$ and $\left(\bar{Q}_{m n}\right)_{k}$ are the transformed reduced stiffnesses and transformed shear stiffnesses of the $k$-th 
layer, and $z_{k-1}$ and $z_{k}$ are defined in Fig. 1. The value of the shear correction factors $K_{m n}$ are taken to be 5/6.

The hygrothermal forces and moments appearing in Eqs. [3] and [4] are defined as

$$
\begin{aligned}
\left(N_{i}^{T M}, M_{i}^{T M}\right) & =\sum_{k=1}^{N} \int_{z_{k-1}}^{z_{k}}(1, z)\left(\bar{Q}_{i j}\right)_{k}\left(\left(\bar{\alpha}_{j}\right)_{k} \Delta T\right. \\
& \left.+\left(\bar{\beta}_{j}\right)_{k} \Delta c\right) \mathrm{d} z \quad(i, j=1,2,6)
\end{aligned}
$$

in which $\left(\alpha_{j}\right)_{k}$ and $\left(\bar{\beta}_{j}\right)_{k}$ are the transformed coefficients of the thermal expansion and transformed swelling coefficients of the $k$-th layer, respectively.

The equations governing the shell responses can be derived using the principle of virtual work. The corresponding energy formulation for the linear and nonlinear analyses is

$$
\begin{gathered}
\delta W=\int_{A}\left(N_{i} \delta \varepsilon_{i}+Q_{m} \delta \varepsilon_{m}+M_{i} \delta \kappa_{i}+\rho \delta w\right) \mathrm{d} A \\
+\int_{S} p R / 2 \delta u \mathrm{~d} S=0 \\
\quad(i=1,2,6 ; m==4,5)
\end{gathered}
$$

in which $\mathrm{d} A$ represents a differential area of the middle surface, and $\mathrm{d} S$ is the differential length along the edge of the middle surface. In arriving at expression [8], the Love geometry assumption (neglecting $z / R$ ) has been invoked.

Next, consider the virtual work formulation for the linear buckling analysis. Let the equilibrium state at the bifurcation point be denoted by

$$
\left(\dot{u}, \bar{v}, \bar{w}, \bar{\theta}_{x}, \bar{\theta}_{y}, \bar{\varepsilon}_{i}, \bar{\varepsilon}_{m}, \bar{\kappa}_{i}, \bar{N}_{i}, \bar{Q}_{m}, \bar{M}_{i}, \bar{p}, \bar{T}, \bar{c}\right)
$$

An infinitesimally adjacent equilibrium position, without changing the loading and hygrothermal conditions, having the following variations from the previous unbuckled equilibrium state are to be determined:

$$
\left(\tilde{u}, \tilde{v}, \tilde{w}, \tilde{\theta}_{x}, \tilde{\theta}_{y}, \tilde{\varepsilon}_{i}, \tilde{\varepsilon}_{m}, \tilde{\kappa}_{i}, \tilde{N}_{i}, \tilde{Q}_{m}, \tilde{M}_{i}\right)
$$

The corresponding virtual work of the system moving from the unbuckled state to a buckled position is

$$
\begin{aligned}
\delta W & =\int_{A}\left[\left(\bar{N}_{i}+\widetilde{N}_{i}\right) \delta\left(\widetilde{\varepsilon}_{i}^{l}+\widetilde{\varepsilon}_{i}^{n l}\right)+\left(\bar{Q}_{m}+\tilde{Q}_{m}\right) \delta \widetilde{\varepsilon}_{m}\right. \\
& \left.+\left(\bar{M}_{i}+\widetilde{M}_{i}\right) \delta \widetilde{\kappa}_{i}+\bar{p} \delta \tilde{w}\right] \mathrm{d} A \\
& +\int_{s} \bar{p} R / 2 \delta \tilde{u} \mathrm{~d} S \quad(i=1,2,6 \quad ; \quad m=4,5)
\end{aligned}
$$

By employing the equilibrium conditions governing the prebuckled state and by neglecting the terms involving higher-order variations of displacements, the mathematical expression of the principle of virtual work can be reduced to

$$
\begin{aligned}
& \int_{A}\left(\tilde{N}_{i} \delta \tilde{\varepsilon}_{i}^{l}+\tilde{Q}_{m} \delta \tilde{\varepsilon}_{m}+\tilde{M}_{i} \delta \tilde{K}_{i}+\overline{N_{i}} \delta \tilde{\varepsilon}_{i}^{n l}\right) \mathrm{d} A=0 \\
& (i=1,2,6 \quad ; \quad m=4,5)
\end{aligned}
$$

where the nonlinear parts $\tilde{\varepsilon}_{i}^{n l}$ of the incremental strains are as follows,

$$
\tilde{\varepsilon}_{x}^{n l}=\left(\tilde{w}_{x}\right)^{2} / 2, \quad \tilde{\varepsilon}_{y}^{n l}=\left(\widetilde{w}_{y}\right)^{2} / 2, \quad \tilde{\gamma}_{x y}^{n l}=\widetilde{w}_{x} \tilde{w}_{y}
$$

while strains and curvatures $\left(\tilde{\varepsilon}_{i}^{l}, \tilde{\varepsilon}_{m}, \tilde{\mathrm{\kappa}}_{i}\right)$, and stress resultants $\left(\tilde{N}_{i}, \bar{N}_{i}, \tilde{Q}_{m}, \tilde{M}_{i}\right)$ appearing in Eq. [9] are calculated according to the small deformation theory.

\section{FINITE ELEMENT FORMULATIONS}

Following the standard finite element procedures, the middle surface of the shell is discretized into $N_{e}$ elements. The deformation variables of an element having $N_{n}$ nodes are approximated as

$$
\left(u, v, w, \theta_{x}, \theta_{y}\right)=\sum_{i=1}^{N_{n}} N_{i}\left(u_{i}, v_{i}, w_{i}, \theta_{x i}, \theta_{y i}\right)
$$

in which $N_{i}$ is the shape function and $\left(u_{i}, v_{i}, w_{i}, \theta_{x i}, \theta_{y i}\right)$ are nodal generalized displacements. The expression of the principle of the virtual work can be expressed as

$$
\delta W=\sum_{i=1}^{N_{e}} \delta W_{i}=0
$$

where $\delta W_{i}$ is the virtual work of an element.

\section{Linear buckling analysis}

For the case of linear buckling analysis, the resulting algebraic equations from Eq. [9] are

$$
\left(K_{i j}+\lambda_{p} K_{i j}^{p}+\lambda_{h} K_{i j}^{h}\right) \tilde{a}_{j}=0
$$

where $\tilde{a}_{j}$ represents the nodal incremental displacement, and parameters $\lambda_{p}$ and $\lambda_{h}$ are the loading parameters which scale the pressure $p$ and changes of the hygrothermal conditions $(\Delta T, \Delta c)$ such that

$$
p=\lambda_{p} \hat{p} \quad, \quad(\Delta T, \Delta c)=\lambda_{h}(\Delta \hat{T}, \Delta \hat{c})
$$


with pressure $\hat{p}$, and $\left[K^{h}\right]$ is corresponding to the hygrothermal conditions $\Delta \hat{T}$ and $\Delta \hat{c}$. Both matrices are calculated from the linear prebuckling analysis. In the present analysis, the parameter $\lambda_{h}$ is set to be equal to one. The buckling pressure $\bar{p}$ is determined by finding the eigenvalue $\lambda_{p}$ of Eqs. [10].

\section{Nonlinear postbuckling analysis}

Application of the finite element procedure to the energy formulation [8] yields the following set of nonlinear algebraic equations :

$$
\begin{aligned}
& \Psi_{i}\left(a, \lambda_{p}, \lambda_{h}\right)=K_{i j}\left(a, \lambda_{p}, \lambda_{h}\right) j \\
& -\lambda_{p} f_{i}^{p}-\lambda_{h}\left(f_{i}^{h}+F_{i j} a_{j}\right)=0
\end{aligned}
$$

where $\mathbf{a}$ is the vector of the nodal displacements, and $\lambda_{p}$ and $\lambda_{h}$ are the loading parameters which scale pressure, temperature's rise and increase of moisture content in the same way as indicated in Eqs. [11]. The function $\Psi_{i}$ appearing in Eqs. [12] is referred to as the unbalanced force function. The solution to the above equations are obtained using an incremental/iterative procedure; that is, the nonlinear problem is stepwise linearized and linearization errors is corrected by additional iterations as described hereafter.

At the $k$-th iteration stage, the unbalanced force function $\Psi_{i}^{k}\left(=\Psi_{i}\left(\mathbf{a}^{k}, \lambda_{p}^{k}, \lambda_{h}^{k}\right)\right)$ can be expanded into a Taylor series around the previous iteration state $\left(\mathbf{a}^{k-1}, \lambda_{p}^{k-1}, \lambda_{h}^{k-1}\right)$ as

$\Psi_{i}^{k}=\Psi_{i}^{k-1}+\frac{\partial \Psi_{i}^{k-1}}{\partial a_{i}} \delta a_{j}^{k}+\frac{\partial \Psi_{i}^{k-1}}{\partial \lambda_{p}} \delta \lambda_{p}^{k}+\frac{\partial \Psi_{i}^{k-1}}{\partial \lambda_{h}} \delta \lambda_{h}^{k}+\ldots$.

where

$a_{i}^{k}=a_{i}^{k-1}+\delta a_{i}^{k}=\mathbf{a}_{i}^{0}+\nabla a_{i}^{k}, \quad \nabla a_{i}^{k}=\nabla a_{i}^{k-1}+\delta a_{i}^{k}$

$\lambda_{p}^{k}=\lambda_{p}^{k-1}+\delta \lambda_{p}^{k}=\lambda_{p}^{0}+\nabla \lambda_{p}^{k}, \quad \nabla \lambda_{p}^{k}=\lambda_{p}^{k-1}+\delta \lambda_{p}^{k}$

$\lambda_{h}^{k}=\lambda_{h}^{k-1}+\delta \lambda_{h}^{k}=\lambda_{h}^{0}+\nabla \lambda_{h}^{k}, \quad \nabla \lambda_{h}^{k}=\lambda_{h}^{k-1}+\delta \lambda_{h}^{k}$

$\operatorname{Here}\left(\mathbf{a}^{0}, \lambda_{p}^{0}, \lambda_{h}^{0}\right)$ is a known equilibrium state; i.e. $\Psi_{i}^{0}=0$.

The linear expansion of Eqs. [13] together with Eqs.

[14] can be used as an iteration scheme to obtain the zero of the unbalanced force $\Psi_{i}$, i.e. the equilibrium state. Hopefully, at the $k$-th iteration stage, the unbalanced force $\Psi_{i}^{k}$ attains a value near zero, in which case the iteration formulation can be written as

$$
\frac{\partial \Psi_{i}^{k-1}}{\partial a_{j}} \delta a_{j}^{k}=-\Psi_{i}^{k-1}-\frac{\partial \Psi_{i}^{k-1}}{\partial \lambda_{p}} \delta \lambda_{p}^{k}-\frac{\partial \Psi_{i}^{k-1}}{\partial \lambda_{h}} \delta \lambda_{h}^{k}
$$

where $\partial \Psi_{i} / \partial a_{j}$ is known as the Jacobian matrix, sometimes referred to as the tangential stiffness matrix.

The conventional Newton-Raphson method uses loading parameters $\lambda_{p}$ and $\lambda_{h}$ as the control parameters. At the incremental stage $(k=1)$, the increment in the displacement $\delta a_{j}^{1}\left(=\nabla a_{j}^{1}\right)$ is computed from Eqs. [15] by specifying variations in loading parameters, i.e. $\delta \lambda_{p}^{1}\left(=\nabla \lambda_{p}^{1}\right)$ and $\delta \lambda_{h}^{1}\left(=\nabla \lambda_{h}^{1}\right)$. The errors in the displacements are corrected using the iteration formulations [15] by setting both $\delta \lambda_{p}^{k}$ and $\delta \lambda_{h}^{k}(k \geq 2)$ equal to zero. This method fails to trace the equilibrium curve when the specified loading is above (or below) the limit point.

The arc-length method has been employed to trace the post-limit equilibrium curve. In this method the length of the equilibrium curve, rather than the loading parameters, is employed as the control parameter. The iteration formulation [15] is constrained by the equation (Ramm, 1981; Crisfield, 1981)

$$
\nabla a_{i}^{k} \nabla a_{i}^{k}+\left(\nabla \lambda_{p}^{k}\right)^{2}+\left(\nabla \lambda_{h}^{k}\right)^{2}=(\nabla S)^{2}
$$

where $\nabla S$ is a prescribed length. Equation [16], called the spherical constraint, not only limits the step length of the iteration but also confines the iteration direction.

Combining Eqs. (15) and (16) leads to a set of $(m+1)$ equations. The $(m+1) \times(m+1)$ square coefficient matrix generally does not exhibit banded or symmetric form. To make use of the symmetric and banded structure of the tangential stiffness matrix, the technique suggested by Ramm (1981) is used.

The details of tracing the postbuckling path of the shells subjected to both external pressure and hygrothermal forces are explained below:

1. First, specify loading parameters such that $\lambda_{p}=0$ and $\lambda_{h}=1$; i.e. $p=0, \Delta \hat{T}=\Delta T$ and $\Delta \hat{c}=\Delta c$. The corresponding equilibrium position is obtained from formulation [15] by applying the Newton-Raphson technique.

2. Based on the previous equilibrium position, use the arc-length technique to trace the pressure-displacement curve based on Eqs. [14] - [16] by assigning $\lambda_{h}=$ 0 and specifying an arc-length $\nabla S$.

3. To overcome the difficulty of searching for a postbifurcation point, a very small concentrated load is placed on the location where maximum deflection occurs. The perturbation is immediately removed once the post-critical position is located. The trace of the postbuckling curve can be executed automatically.

\section{NUMERICAL EXAMPLES}

The structural responses of Glass/Epoxy circular cylindrical shells are reported hereafter. The application of pressure and hygrothermal loadings is assumed to be timewise constant. The FRP material is assumed to be a 
hygrothermorheologically simple material (HTSM). The effects of temperature's rise and the increase of moisture concentration on the creep behavior are accommodated in the constitutive equation by the use of "reduced time" (Maksimov et al. , 1975 ; Weitsman, 1977). Within the framework of quasi-elastic analysis, the stress-strain relations for a lamina are

$$
\sigma_{i}(t)=\bar{Q}_{i j}(\xi(t)) \varepsilon_{j}(t) \quad(i, j=1,2,6)
$$

and

$$
\sigma_{m}(t)=\bar{Q}_{m n}(\xi(t)) \varepsilon_{n}(t) \cdot(m, n=4,5)
$$

where $\xi(t)$ is the "reduced time" which is related to the real time and the combined temperature-moisture shift factor $a_{T M}(T(t), c(t))$ as follows,

$$
\xi(t)=\int_{0}^{t} \frac{\mathrm{d} t^{\prime}}{a_{T M}\left(t^{\prime}\right)}
$$

It is obviously that $\xi(t)=t / a_{M M}$ if the variations of temperature and moisture are time-independent. The combined shift function $a_{T M}$ for this Glass/Epoxy is assumed to be the product of the temperature shift factor $a_{T}(T(t))$ and the moisture shift factor $a_{M}(c(t))$, namely

$$
a_{T M}=a_{T} a_{M}
$$

The values of $a_{T}$ and $a_{M}$ are assumed as follows,

$$
a_{T}=\exp (45.81-0.1324 T), a_{M}=\exp (0.26-68.73 c)
$$

where $T$ is absolute temperature in Kelvin scale and $c$ is the specific moisture concentration.

The creep compliances of Glass/Epoxy lamina, measured at approximately $73^{\circ} \mathrm{C}$ degree and $21 \%$ relative humidity, are (Lou and Schapery, 1971) :

$$
\begin{aligned}
& S_{11}=0.0267 S_{0}, S_{12}=-0.00826 S_{0}, \\
& S_{22}=0.0942\left(1+0.0846 t^{0.27}\right) S_{0}, \\
& S_{55}=S_{66}=0.233\left(1+0.151 t^{0.27}\right) S_{0}, \\
& S_{44}=3 S_{66}
\end{aligned}
$$

where $S_{0}$ represents a reference value of $(\mathrm{GPa})^{-1}$, and the values of $S_{44}$ and $S_{55}$ are assumed. The coefficients of thermal expansion and swelling coefficients of an orthotropic lamina are (Tsai and Hahn, 1980) :

$\alpha_{1}=8.6 \times 10^{-6} /{ }^{\circ} \mathrm{C}, \alpha_{2}=22.1 \times 10^{-6} /{ }^{\circ} \mathrm{C}, \beta_{1}=0.0, \beta_{2}=0.44$

The equilibrium moisture concentration $c$ is related to the relative humidity $M$ of the environment by the following law (Tsai and Hahn, 1980) :

$$
c=0.018\left(\frac{M}{100}\right)
$$

The shell is assumed to be free of any hygrothermal strain in an environment with $T=23^{\circ} \mathrm{C}$ and $M=21 \%$. The operating condition of the shell is $T=73^{\circ} \mathrm{C}$ and $M=96 \%$. The cylindrical shells considered have simply-supported edges. The specific boundary conditions on edges $x=0, L$ are as follows,

$$
N_{x}=p R / 2, v=w=M_{x}=\theta_{y}=0
$$

The nine-node Lagrangian elements are exclusively used in this study. The selective reduced integration is employed to integrate the elemental energy density. The extensional, coupling and bending strain energies are calculated using the $3 \times 3$ Gaussian quadrature (three points in each direction ). The integration of transverse shear strain energy is performed using the $2 \times 2$ scheme. For the cases of symmetric and antisymmetric cross-ply cylinders, a quadrant of a buckled wave needs to be discretized. For the cases of angle-ply cylinders, the whole domain of a buckled wave needs to be considered. The results reported hereafter are based on the $3 \times 8$ mesh for crossply cylinders and the $6 \times 16$ mesh for angle-ply cylinders.

EXAMPLE 1 :Buckling Pressure of Angle-Ply Cylinders

$$
\left((\theta /-\theta)_{4}, L / R=4, R / h=50\right)
$$

The viscoelastic buckling of the eight-layer, regular antisymmetric angle-ply cylindrical shells with geometric aspect ratios $R / h=50, L / R=4$ and having various angles of fiber reinforcement is considered. The critical pressure is obtained using the linear classical analysis. The reduction of the initial buckling pressures corresponding to various loading durations are shown in Fig. 2. The elastic buckling pressures $\bar{p}_{0}$ (loading duration $t=0$ ) for the shells having different laminations are indicated below the figure's caption. It is noted from Fig. 2 that the creep effect on the buckling pressure is very significant, especially for the laminates having a smaller angle of fiber-reinforcement. For example, the initial buckling pressure for an axially reinforced $\left(\theta=0^{\circ}\right)$ shell with pressure duration $t=10^{5}$ hours is only approximately onethird of the elastic buckling pressure $(t=0)$ of the same shell. The buckling configuration of a shell with $\theta=67.5^{\circ}$ and loading duration $t=10^{5}$ hours is shown in Fig. 3. It is seen that the cylinder buckles into a three-wave shell and its nodal lines are not parallel to the longitudinal axis.

EXAMPLE 2 :Postbuckling of a Cross-Ply Cylinder 


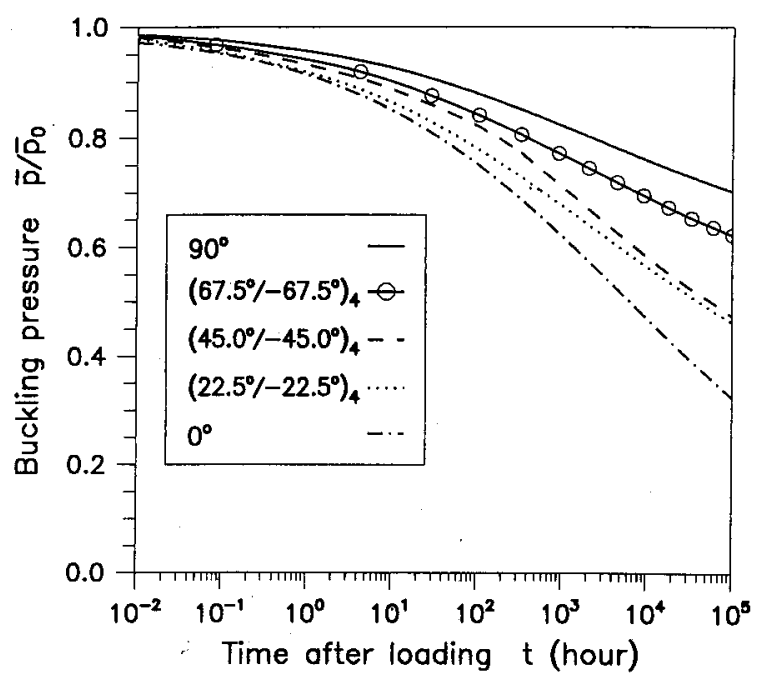

Fig. 2. Reduction in bucking pressures of angle-ply cylinders $(L / R=4$, $R h=50$ ) corresponding to various loading durations.

$\begin{array}{ll}\theta=90^{\circ} & : \bar{p}_{0}=0.277 \mathrm{MPa}, \\ \left(67.5^{\circ} /-67.5^{\circ}\right)_{4} & : \bar{p}_{0}=0.257 \mathrm{MPa}, \\ \left(45.0^{\circ} /-45.0^{\circ}\right)_{4} & : \bar{p}_{0}=0.214 \mathrm{MPa} \\ \left(22.5^{\circ} /-22.5^{\circ}\right)_{4} & : \bar{p}_{0}=0.172 \mathrm{MPa}, \\ \theta=0^{\circ} & : \bar{p}_{0}=0.161 \mathrm{MPa} .\end{array}$

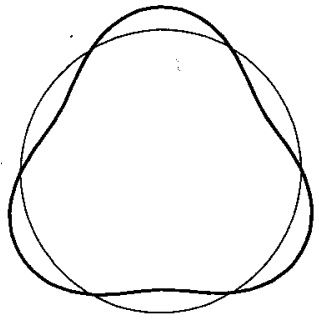

(a)

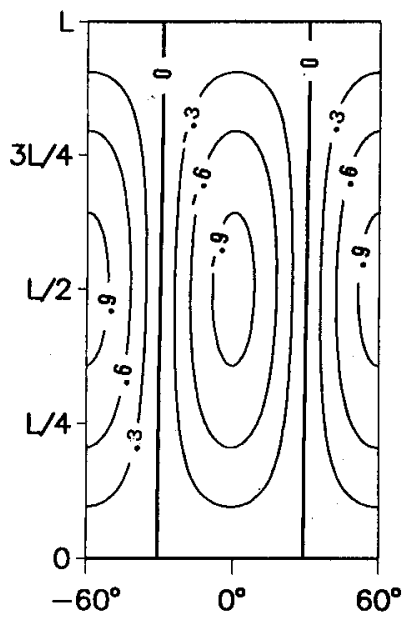

(b)

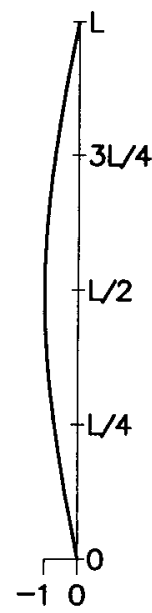

(c)
Fig. 3. Buckling deflection ( $\left.w / w_{\max }\right)$ of an eight-layer antisymmetric angle-ply cylinder $\left(\theta=67.5^{\circ}, L R=4, R / h=50\right)$.

(a): Deflection shape on $x=L / 2$.

(b): Deflection contour of a buckled wave.

(c): Deflection shape on $y=0$.
The relations between external pressure and maximum inward deflection of the eight-layer, regular crossply elastic shell (assuming pressure duration $t=0$ ) exposed to the hygrothermal environment $\left(\Delta T=50^{\circ} \mathrm{C}, \Delta c=0.0135\right)$ is shown in Fig. 4. This figure includes the possible equilibrium curves of the shell having four, five and six circumferential buckled waves. For a geometrically perfect shell, the finite element procedure predicts that the cylinder will snap into a five-wave shell as pressure $p$ reaches above $0.508 \mathrm{MPa}$. The snap-through point is indicated by $S^{\prime}$. It is noted from Fig. 4 that the lowest postbuckling equilibrium pressure for a shell having five buckled waves is approximately $p=0.392 \mathrm{MPa}$, and that the lowest postbuckling equilibrium pressure for a shell having four buckled waves is $p=0.260 \mathrm{MPa}$. This suggests the snap-through can occur at a much lower pressure for a geometrically imperfect shell.

The equilibrium paths of the cross-ply shell corresponding to loading duration $t=10^{5}$ hours is shown in Fig. 5. This figure shows that for a perfect shell under pressure $p=0.285 \mathrm{MPa}$ and the prescribed hygrothermal conditions, the shell will snap into a new equilibrium position $S$ ' as loading duration exceeds $10^{5}$ hours. The buckled shell has four waveforms with its deflection configuration depicted in Fig. 6. A further examination of Fig. 5 reveals that the corresponding lowest postbuckling pressures for a shell having four and five buckled waves are $0.193 \mathrm{MPa}$ and $0.138 \mathrm{MPa}$, respectively.

By examining the postbuckling behaviors of this particular cross-ply shell (Figs. 4 and 5), it is concluded that, for an imperfect, viscoelastic shell under a long term service ( $10^{5}$ hours ), the buckling pressure can be as

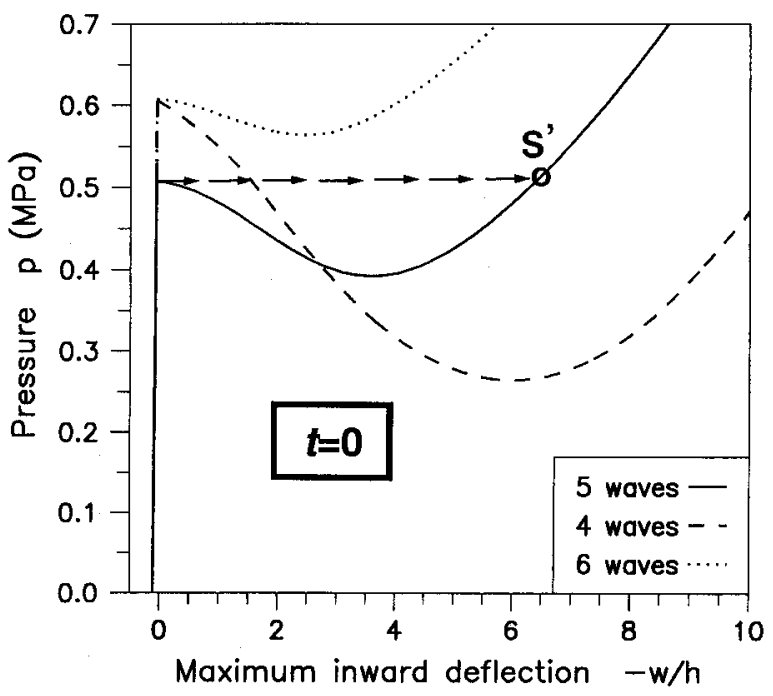

Fig. 4. Relations between externalpressure and maximum inward deflection of a cross-ply cylin der $\left(\left(0^{\circ} / 90^{\circ}\right)_{4}, L R=2, R h=50\right)$ corresponding to loading duration $t=0$. 


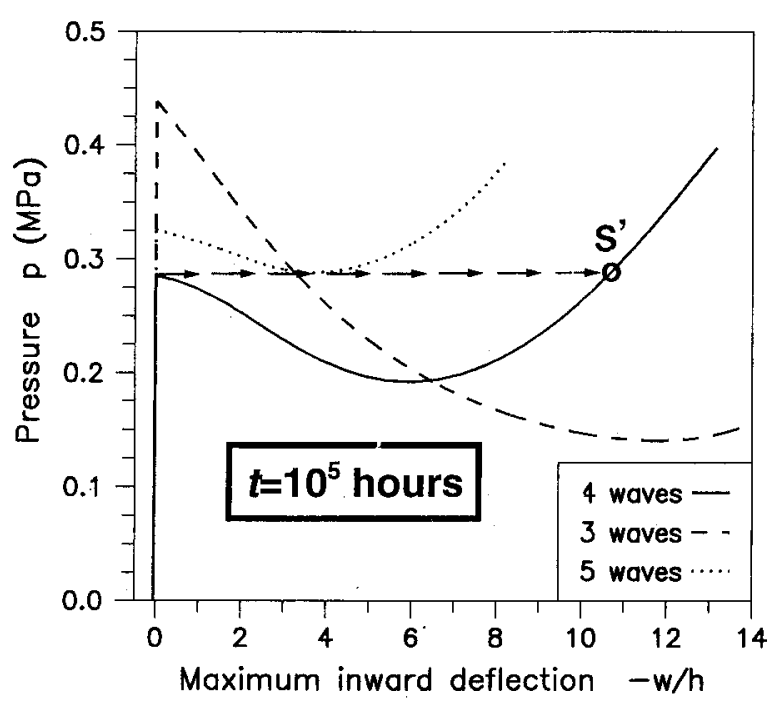

Fig. 5. Relations between external pressure and maximum inward deflection of a cross-ply cylinder $\left(\left(0^{\circ} / 90^{\circ}\right)_{4}, L / R=2, R / h=50\right)$ corresponding to loading duration $t=10^{5}$ hours.

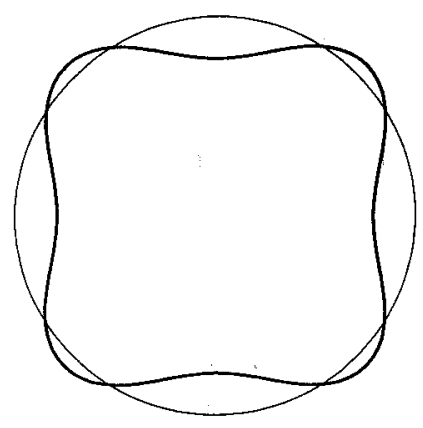

(a)

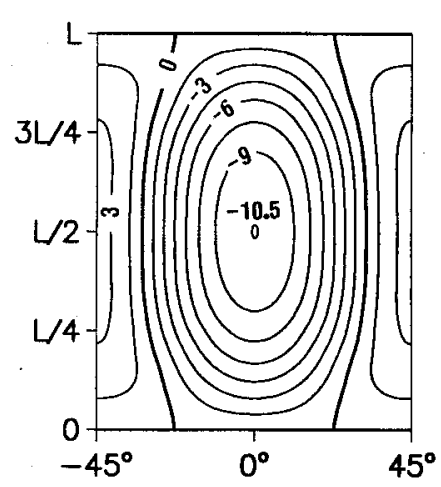

(b)

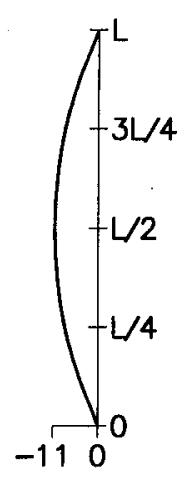

(c)
Fig. 6. Postbuckling deflection $w$ of a cross-ply cylinder $\left(\left(0^{\circ} / 90^{\circ}\right)_{4}\right.$, $L R=2, R h=50$ ) with loading duration $t=10^{5}$ hours.
(a): Deflection shape on $x=L / 2$.
(b): Deflection contour of a buckled wave.
(c): Deflection shape on $y=0$.

low as less than one third $(0.138 \mathrm{MPa} / 0.508 \mathrm{MPa})$ of the initial buckling pressure associated with a shell considered to be geometrically perfect and purely elastic.

EXAMPLE 3 :Postbuckling of an Angle-Ply Cylinder

$$
\left(\left(45^{\circ} /-45^{\circ}\right)_{4}, L / R=2, R / h=50\right)
$$

The relations between external pressure and maximum inward deflection of the angle-ply shell corresponding to pressure durations $t=0$ and $10^{5}$ hours are shown in Figs. 7 and 8, respectively. Figure 7 indicates that for an elastic shell $(t=0)$ the initial buckling pressure and the lowest postbuckling equilibrium pressure are 0.452 $\mathrm{MPa}$ and $0.250 \mathrm{MPa}$, respectively. For the case of a viscoelastic shell under $10^{5}$ hours service, it is observed from Fig. 8 that the initial critical pressure and the lowest postbuckling pressure of the shell are reduced to 0.260 $\mathrm{MPa}$ and $0.175 \mathrm{MPa}$, respectively.

\section{CONCLUDING REMARKS}

The quasi-elastic analysis of buckling and postbuckling behaviors of hygrothermoviscoelastic FRP cylindrical shells has been conducted using the finite element technique. The numerical results for the Glass/ Epoxy shells show that the viscoelastic effect on the critical pressure is very significant. A shell sustains a much lower pressure without buckling during a long term service. The postbuckling analysis, based on the instant snap-through concept, reveals that the practically unavoidable imperfection can further reduce the resistance of shells to buckling. The minimum postbuckling equilibrium pressure can be considered as the lower limit for the buckling load.

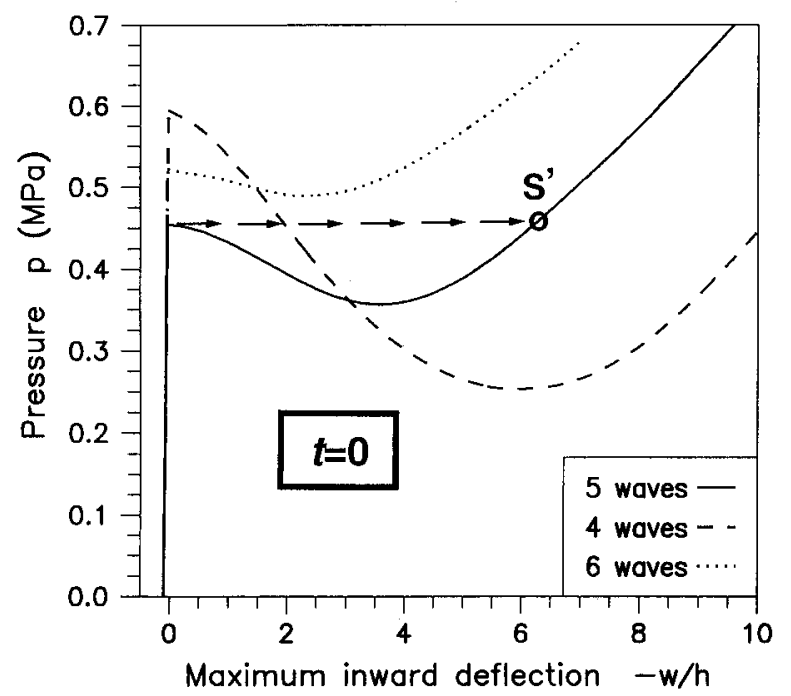

Fig. 7. Relations between external pressure and maximum inward deflection of an angle-ply cylinder $\left(\left(45^{\circ} /-45^{\circ}\right)_{4}, L R=2, R h=50\right)$ corresponding to loading duration $t=0$. 


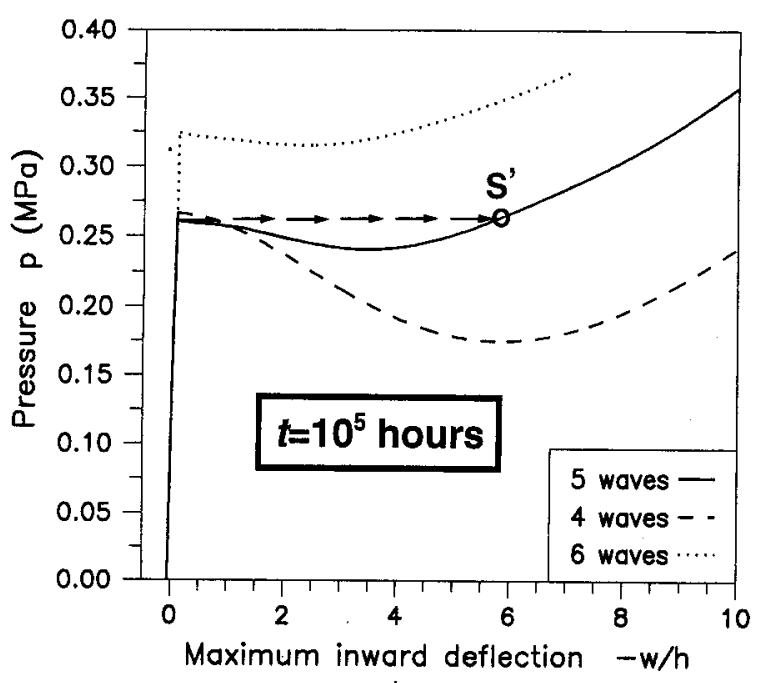

Fig. 8. Relations between external pressure and maximum inward deflection of an angle-ply cylinder $\left(\left(45^{\circ}-45^{\circ}\right), L R=2, R h=50\right)$ corresponding to loading duration $t=10^{5}$ hours.

\section{ACKNOWLEDGMENTS}

The support of this research by the National Science Council of Taiwan, R.O.C. through Grant NSC82-0401E-019-18 is gratefully acknowledged.

\section{REFERENCES}

1. Baker, E.H. (1968). Introduction to the theory of shells. ShellAnalysis Manual (edited by A.P.Cappelli),

\section{1-165, NASA CR-912.}

2. Chang, J.S. and Cheng C.H. (1991). Viscoelastic buckling of cross ply laminated cylindrical circular shells based on a high order displacement field. The Chinese Journal of Mechanics 7 (2), 99-107.

3. Crisfield, M.A. (1981). A fast incremental/iterative solution procedure that handles snap-through. Computers \& Structures 13, 55-62.

4. Lou, Y.C. and Schapery, R.A. (1971). Viscoelastic characterization of a nonlinear fiber-reinforced plastic. J. Composite Materials 5, 208-234.

5. Maksimov, R.D., Sokolov, Y.A. and Mochalov, V.P. (1975). Effect of temperature and humidity on creep of polymer materials. Mechanica Polimerov, 393399.

6. Ramm, E. (1981). Strategies for tracing the nonlinear response near limit point. Nonlinear Finite Element Analysis in Structural Mechanics (edited by W. Wunderlich, E. Stein and K. J. Bathe), 63-89.

7. Tsai, S.W. and Hahn, H.T. (1980). Introduction to Composite Materials. Technomic Publishing, Westport, CT.

8. Weitsman, Y. (1977). Effects of fluctuating moisture and temperature on the mechanical response of resinplates. J. Applied Mechanics 44, 571-576.

9. Wilson, D.W. and Vinson, J.R. (1984). Viscoelastic analysis of laminated plate buckling. AIAA Journal 22, 982-988.

10. Yamaki, N. (1984). Elastic Stability of Circular Cylindrical Shells. Elsevier, Amsterdam. 